\title{
First- and second-order configural sensitivity for greeble stimuli in baboons
}

\author{
Carole Parron and Joël Fagot \\ LPC, UMR 6146, CNRS, University of Provence, Marseille, France
}

\begin{abstract}
Previous studies on nonhuman primates have shown inconsistencies in their processing of first- and secondorder relational properties of facial stimuli. Using greeble stimuli sharing configural properties with faces, this study assessed configural processing in baboons. Five baboons were trained to recognize a positive stimulus among pairs of greebles in a two-alternative forced choice task. They were then tested with new stimulus pairs involving either a first-order version, with modifications in global qualitative spatial relations, or a second-order version, with modifications of finer spatial relations. Performance remained above chance in all test conditions, including when only second-order cues were available, but it was higher for first-order trials. It is proposed that an extensive training with greebles led to the processing of second-order relational properties. These results demonstrate that configural sensitivity is not restricted to faces in baboons and suggest that a common mechanism may support configural processing for face and nonface stimuli.
\end{abstract}

An impaired recognition of upside-down faces, known as the inversion effect (e.g., Yin, 1969), is traditionally studied to investigate configural processing. In humans, reliance on configural cues is disrupted after facial inversion, yielding to a featural analysis of the inverted faces (Maurer, Le Grand, \& Mondloch, 2002). In nonhuman primates, inspection of the literature reveals a debate on the importance of featural and configural cues for the processing of facial stimuli, due to inconsistent effects of face inversion. On one hand, macaques and chimpanzees have been found to express no effect of inversion for conspecific faces presented in a forced choice task (e.g., Bruce, 1982; Rosenfeld \& van Hoesen, 1979; Tomonaga, Itakura, \& Matsuzawa, 1993). On the other hand, other studies have suggested that upright faces are processed differently from upside-down ones in both macaques (Tomonaga, 1994) and chimpanzees (Parr, Dove, \& Hopkins, 1998), as is the case with humans (Maurer et al., 2002). These conflicting results suggest at least that, depending on the experimental context, nonhuman primates may attend both featural and configural information. For instance, familiarity with the stimulus has been shown to play an important role in configural processing. Inversion effects have been successfully obtained with sets of nonface objects (e.g., dogs of the same breed, Diamond \& Carey, 1986; caricatures of birds, Rhodes \& McLean, 1990) for which human subjects were expert. Face recognition might thus involve mechanisms specialized for the processing of object classes other than faces for which an individual has expertise (Bukach, Gauthier, \& Tarr, 2006). In humans, sensitivity to configural cues has also been demonstrated with greebles: artificial stimuli rendered in three dimensions with configural properties (see Figure 1). Gauthier and Tarr (1997) and Gauthier, Williams, Tarr, and Tanaka (1998) reported that subjects with expertise with greebles showed processing of configural cues as they did when tested with faces.

From a theoretical point of view, a discussion of configural processing within faces should consider the distinction between first- and second-order configural properties. First-order refers to the global qualitative spatial relations among facial features (e.g., the eyes are above the nose), whereas second-order refers to the fine spatial relations among features (e.g., the distance between the nose and the mouth relative to the prototypical face arrangement; Diamond \& Carey, 1986). To our knowledge, only three studies have compared the processing of first- and secondorder configural cues within faces in nonhuman primates. Parr, Heintz, and Akamagwuna (2006) created three kinds of facial stimuli. One preserved both first- and secondorder relational features (inner features extracted). The second preserved first-order cues but impaired secondorder relations (features fractured apart). The third had both first- and second-order cues altered (features rearranged). The results showed recognition impairments only when second-order cues were altered. Parr, Heintz, and Pradhan (2008, Experiment 2) later used a similar procedure in macaques. Their performance was impaired after both first- and second-order manipulations, but also when these cues were preserved, suggesting no particular reliance on configural cues. According to the authors, macaques may have relied on external facial features to solve the task, and these features were absent in the test trials. The last study (Parron \& Fagot, 2007a) tested in baboons 


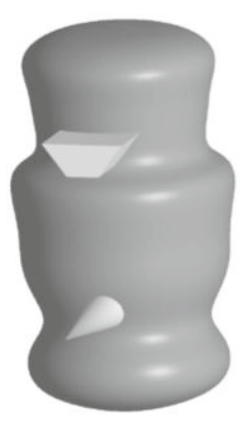

A

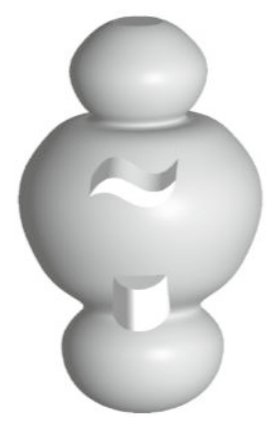

$\mathrm{F}$

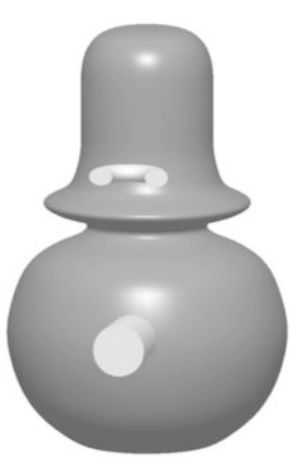

B

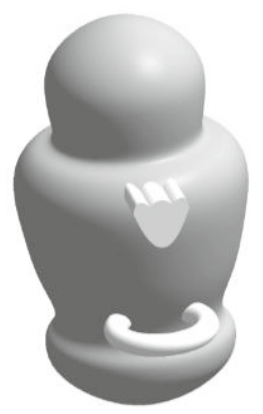

G

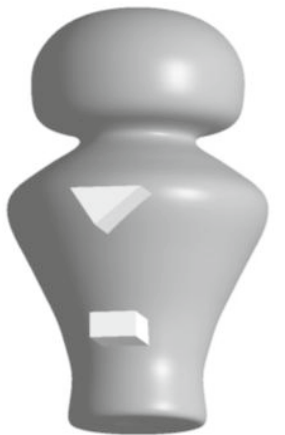

C

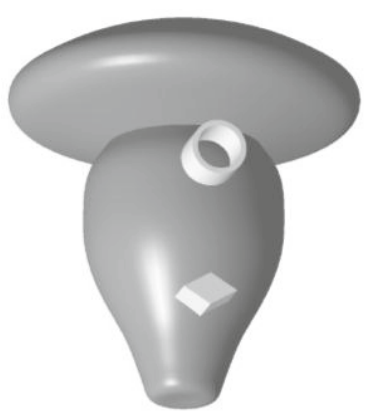

$\mathrm{H}$

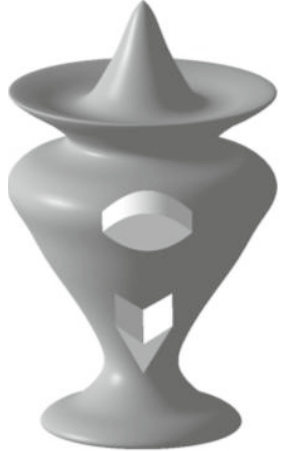

D

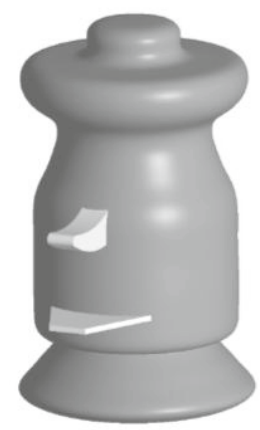

I

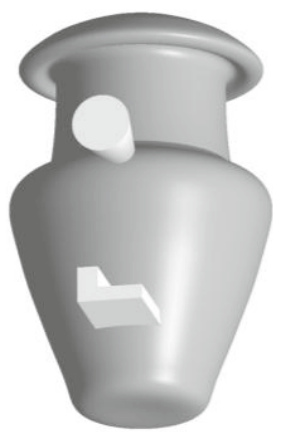

E

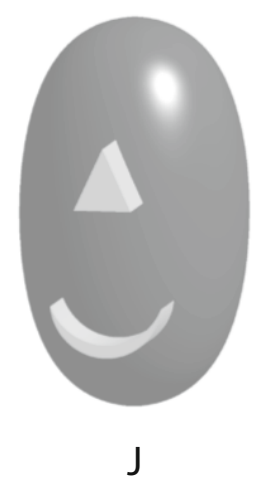

Figure 1. Illustration of the 10 positive greebles $(\mathbf{A}-\mathbf{J})$. Each greeble has a different global shape and two protruding parts on its vertical axis.

the processing of first- and second-order cues within facial stimuli with identical contours. The first transformation implied a reversal of the location of the eyes and the mouth within the face, to change the first-order configural properties of the face. The second transformation, traditionally applied to facial stimuli (e.g., Thompson, 1980), implied an upside-down rotation of the mouth and the eyes relative to the normal face. That alteration of the stimuli meets the definition of second-order configural changes. The baboons failed to spontaneously discriminate a normal face from its second-order modified version but succeeded perfectly in discriminating a normal face from its first-order version. Parron and Fagot (2007a) concluded from these results that, for baboons, first-order properties are more salient than second-order cues.

The present experiment investigated whether baboons would be able to process first- and second-order relational properties within nonface stimuli such as greebles. To our knowledge, this question has never been assessed in monkeys, and it raises another fundamental issue: the specificity of the cognitive processes that monkeys use to identify faces. The use of greebles - simple artificial stimuli sharing configural cues with faces - is helpful in many ways. First, greebles are artificial stimuli with physical properties that can be controlled precisely. Second, given their artificial nature, they are novel objects for the subjects, thus limiting effects of past experience on recognition.
Finally, use of these nonfacial stimuli that share properties with faces may help to disentangle the processing of configural properties from the complex problem of face recognition. In our study, baboons were initially trained to discriminate a positive greeble $(\mathrm{S}+)$ from a negative one $(\mathrm{S}-)$ that differed in terms of both first- and secondorder configural cues. They were then tested with displays contrasting the original $\mathrm{S}+$ stimuli with either a first- or a second-order configural modification of that stimulus serving as $\mathrm{S}-$. The processing of second-order cues was tested with two kinds of modifications: increasing or decreasing the distance between the considered features, using their locations within the original stimulus as reference. We used these conditions to assess whether reliance on second-order cues is scale dependent. The results confirm the ability of baboons to process both first- and second-order configural cues, irrespective of the distance between these features.

\section{METHOD}

\section{Subjects}

Two groups of 20-year-old Guinea baboons (Papio papio) were tested, one comprising 3 males (B03, B05, B07) and one comprising 2 females (B06, B08). Both groups lived in large $(6 \times 4 \mathrm{~m})$ indoor enclosures connected to similarly large outdoor enclosures within the CNRS (Marseille) facility. These baboons had previously been tested in a variety of computerized tasks that required 
the use of a joystick, including two-alternative forced choice (2AFC) experiments (e.g., Deruelle, Barbet, Dépy, \& Fagot, 2000; Parron $\&$ Fagot, 2007a). They had also been involved in two experiments using the same automatic touch-screen system used here (Fagot, Bonté, \& Parron, 2009; Fagot \& Parron, 2010). Each baboon had a $2.1 \times 8 \mathrm{~mm}$ subcutaneous glass tag implanted in each forearm for automatic identification during testing. The use and care of these animals in this research was fully approved by the Comite d'Ethique Régional de la Région Provence-Alpes-Côtes d'Azur.

\section{Apparatus}

The test employed the automatic learning device for monkeys (ALDM), which is described in detail in Fagot and Paleressompoulle (2009). One of two identical ALDM systems was provided ad lib to each social group of baboons. In brief, the test system consisted of a freely accessible test chamber $(70 \times 70 \times 80 \mathrm{~cm})$ in which the rear side was kept open. It was made with waterproof opaque Perspex material, permitting stimulus presentation in well-standardized conditions, irrespective of external weather fluctuations and lighting. Except for the screen, all test equipment was concealed from view and was inaccessible to the experimental subject. The test chamber was fitted in its innermost front side with a $7 \times 7 \mathrm{~cm}$ view port and two $8 \times 5 \mathrm{~cm}$ hand ports. Looking through the view port allowed vision of a 17-in. LCD touch monitor, with a resolution of $1,024 \times$ 768 pixels, installed at eye level, $25 \mathrm{~cm}$ from the view port. Introducing a hand through a hand port gave access to the touch screen. Two antennas fixed around each view port automatically read the radio-frequency identification (RFID) microchip of each subject when it introduced its forearm through the view port. Radio identification signals from the arm tags served to trigger the computer- controlled stimulus presentation and to assign behavioral measures (stimulus choices and response times [RTs]) to each subject. Grains of Ebly dry wheat cereal (www.ebly.co.uk/an/indexfra.htm) served as rewards and were delivered automatically inside the test booth by a homemade food dispenser. The research was controlled by a test program developed by the second author with E-Prime software (V2 professional, Psychology Software Tools, Pittsburgh, PA). That program allows independent test regimens for each individual, irrespective of the order in which baboons presented themselves in the test booth. To prevent the dominant animals from monopolizing the test chambers, a time-out of 15-30 min was imposed on each subject after each session of training or testing trials. That time-out facilitated a turnover within the test systems.

\section{Stimuli}

Stimuli were photorealistically rendered 3-D objects, called greebles (see Gauthier \& Tarr, 1997), as illustrated in Figure 1. They were created with a Catia V5R15 Dassault System, with a resolution of 200 dpi. All greebles had two light-gray protruding parts located along their vertical axis. Pairs of stimuli were presented in each display. The positive stimulus $(\mathrm{S}+)$ of each pair was selected from a set of 10 greebles with two protruding parts (see Figures $1 \mathrm{~A}-1 \mathrm{~J}$ ). In the training session, the negative stimulus $(\mathrm{S}-$ ) of each display was a modified version of $\mathrm{S}+$, with altered first- and second-order properties (see Figure 2A). In the test sessions, $\mathrm{S}-$ alterations affected either the relative position of the two protruding parts (first-order [FO] configural modification, Figure $2 \mathrm{~B}$ ) or the distance between these two parts (second-order configural modification, Figures $2 \mathrm{C}$ and 2D). Note that the contours of the stimuli remained constant in the test pairs. This is justified by earlier demonstrations that the

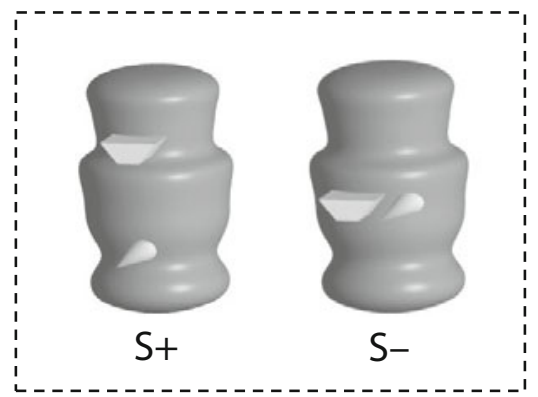

A: Training Session

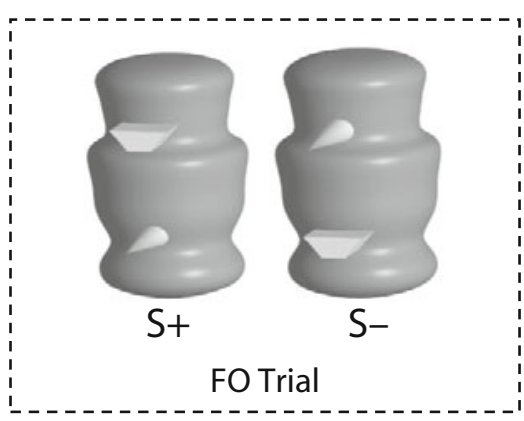

B

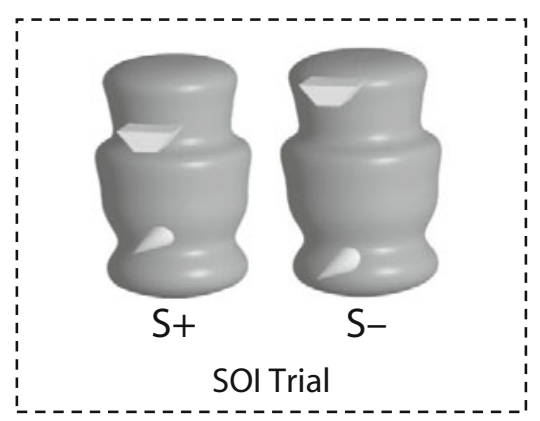

C

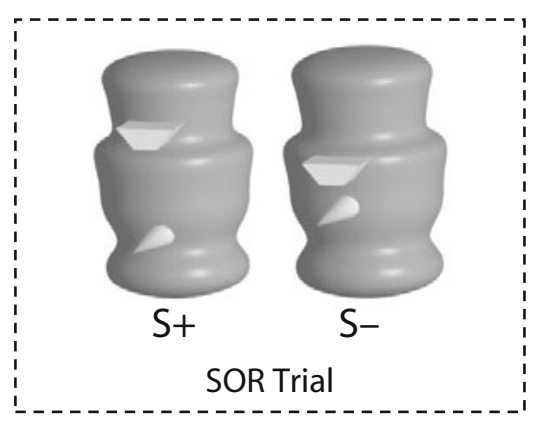

D

\section{Test Sessions}

Figure 2. Illustration of $S+$ and $S-$ from Pair A used during training and testing. Training trial: $S+$ and $S-$ differed in terms of both first- and second-order properties (panel A). Test sessions: first-order (FO) trial (panel B), second-order increased (SOI) trial (panel C), second-order reduced (SOR) trial (panel D). 
contours of faces or objects are salient cues for discrimination in both baboons and macaques (e.g., Martin-Malivel \& Fagot, 2001; Parron \& Washburn, 2010). When the distance was changed, distance between the two protruding parts was increased (Figure 2C) or reduced (Figure 2D), resulting in two subconditions of second-order modifications: the second-order increased (SOI) and second-order reduced (SOR) conditions.

The amount of pixels modified between $\mathrm{S}+$ and its corresponding $\mathrm{S}-$ version was controlled and was maintained to be as similar as possible to our test conditions. On average, over the 10 pairs of stimuli, the first-order modification altered $2.28 \%(S E M=0.49 \%)$ of the pixels of the original S+ stimulus. The SOI and SOR versions of $\mathrm{S}+$, respectively altered $2.58 \%(S E M=0.69 \%)$ and $1.96 \%$ $(S E M=0.62 \%)$ of these pixels. All stimuli measured from 7.2 to $10.3 \mathrm{~cm}\left(13.7^{\circ}-19.5^{\circ}\right.$ of visual angle in our viewing conditions $)$ in width and from 11.5 to $13.5 \mathrm{~cm}\left(21.7^{\circ}-25.3^{\circ}\right)$ in height. Their total surface ranged from 66.9 to $86.9 \mathrm{~cm}^{2}$. The grayscale of each greeble was approximately similar. It ranged from 120 to 182 , on a $256-$ unit scale $(M=147, S E M=5)$. The stimuli were displayed with a $1,024 \times 768$ resolution on a white background.

\section{Procedure}

A $2 \mathrm{AFC}$ procedure was used. Each trial began with the presentation of a fixation stimulus (a $5 \times 5 \mathrm{~cm}$ red cross) in the center of the screen. Touching that stimulus elicited the simultaneous presentation of $\mathrm{S}+$ and $\mathrm{S}-$, with each stimulus displayed randomly on the left or on the right of the screen (see Figure 2A). The task was to recognize and touch $\mathrm{S}+$ on the screen. The stimuli remained on the screen as long as the monkey responded. However, trials with RTs greater than $15 \mathrm{sec}$ were systematically neglected for data analyses because they might reflect inattention or a monkey change within the test system. Correct selections of S + were reinforced by a food reward paired with a melodic tone. By contrast, incorrect responses were followed by a 3 -sec time-out during which the screen turned green and a buzz tone sounded. An intertrial interval of $3 \mathrm{sec}$ minimum was imposed between two trials. After that interval, the next trial was elicited only after the baboon self-identified by introducing one arm in the arm port. The baboons received 10 blocks of trials using the 10 stimuli shown in Figure 1 as $\mathrm{S}+$. Each block consisted in a first training phase requiring identification of $\mathrm{S}+$, followed by a test period. Testing sessions in each block were of three kinds: FO, SOI, and SOR sessions. In FO test sessions, $\mathrm{S}-$ was the first-order modified version of $\mathrm{S}+$ with the location of its protruding parts reversed in comparison with $\mathrm{S}+$. In $\mathrm{SOI}$ test sessions, $\mathrm{S}-$ was the secondorder modified version of $\mathrm{S}+$ for which the distance between the two protruding parts has been expanded. The SOR sessions involved an $\mathrm{S}-$ with an interelement distance reduced in comparison to $\mathrm{S}+$. Each individual received six series of 120 trials for each FO, SOI, and SOR test condition. The baboons therefore received 21,600 trials each, corresponding to six 120-trial sessions per test condition (FO, SOI, SOR) and S + image $(N=10)$. The 120-trial sessions of each test condition each contained 100 baseline trials identical to those used in training (see below), randomly intermixed with 20 probe trials, in which the baboons had to discriminate $\mathrm{S}+$ from its modified test version (see Figure 2B-2D). Within a test session, the probe trials were randomly reinforced on an $80 \%$ basis, irrespective of the performance of the baboon, in order to maintain the animal's motivation and to prevent extinction of its participation, whereas the baseline trials were reinforced only in case of correct $\mathrm{S}+$ recognition. For each stimulus, the six FO, SOI, or SOR test sessions were run in succession. These three conditions were, however, presented in a random order for each image and monkey. The test with a new image was introduced only after the test with the previous image had been completed.

Training trials were run prior to each series of six FO, SOI, and SOR test sessions. Training sessions used the same $\mathrm{S}+$ as in testing but a different $\mathrm{S}-$. The $\mathrm{S}-$ training stimuli differed from $\mathrm{S}+$ in both first- and second-order configural cues (see Figure 2A). First- and second-order configural changes in $\mathrm{S}-$ were aimed at preventing baboons from favoring one or the other kind of cues in training. Training sessions consisted of 100 trials with the left/right location of S+ counterbalanced. They were repeated until the subjects met a criterion of at least $80 \%$ correct in two consecutive training sessions. The program automatically switched to testing once that criterion was met.

\section{RESULTS}

Figure 3 illustrates the mean number of trials required to discriminate the $10 \mathrm{~S}+$ greebles during each training phase $(M=492, S E=163)$. These means were compared with a one-way ANOVA to verify whether some pairs required more training trials than others did. This analysis revealed no significant difference between the 10 pairs $[F(9,36)=1.48, p>.05]$, suggesting that these pairs were of equal difficulty to learn, in spite of the apparent variations, which are shown in Figure 3.

The baboons continued to perform well on the task (percent correct, $M=95.9 \%, S E=0.5$ ) when the training stimuli were represented as baseline trials in the test sessions, showing that the introduction of the probe trials did not disrupt their performance during testing. All the baboons responded above chance $(50 \%)$ in each test condition (one-tailed binomial tests; see Table 1). The unique exception was baboon B06, who was at chance level in the SOR condition. However, accuracy declined for the three types of test stimuli, in comparison with baseline trials (FO, $M=78.31 \%, S E=3.31$; SOI, $M=73.42 \%, S E=$ 4.21; SOR, $M=66.07 \%, S E=2.86)$.

The study of this performance decrement in probe trials considered each test session composed of 20 probe trials mixed with 100 baseline trials. In order to avoid a possible statistical bias due to the larger number of baseline trials that could enhance performance in baseline trials, the next analyses compared the scores of the test conditions (FO, SOR, SOI) with the scores of both the early baseline trials (120 first baseline trials of each session; $M=93.38 \%$ ) and the late baseline trials (120 last baseline trials of each session; $M=96.97 \%$ ). Two three-way test condition (early baseline/late baseline, FO, SOI, SOR) $\times$ $\mathrm{S}+$ identity $(n=10) \times \operatorname{session}(n=6)$ ANOVAs were conducted for that purpose.

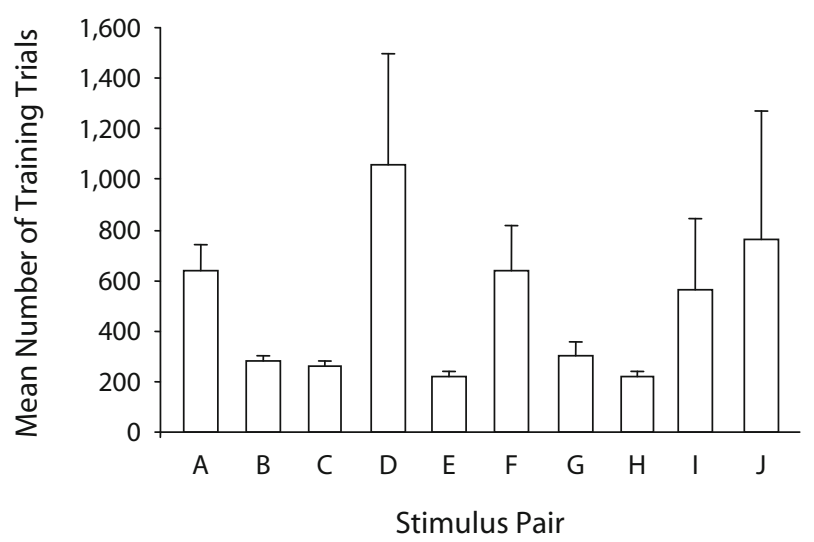

Figure 3. Mean number of training trials (with standard errors) to achieve the training criterion with each stimulus pair. 
Table 1

\begin{tabular}{lccc}
\multicolumn{5}{c}{$\begin{array}{c}\text { Mean Percentages of Correct Responses for the } \\
\text { Five Baboons in Each Testing Condition }\end{array}$} \\
\hline & FO & SOI & SOR \\
\hline B03 & $85.42 \%$ & $72.22 \%$ & $69.00 \%$ \\
& $(z=7.08 ; p<.001)$ & $(z=4.44 ; p<.001)$ & $(z=3.8 ; p<.001)$ \\
B05 & $84.50 \%$ & $75.20 \%$ & $78.33 \%$ \\
& $(z=6.9 ; p<.001)$ & $(z=5.04 ; p<.001)$ & $(z=5.67 ; p<.001)$ \\
B07 & $72.58 \%$ & $82.22 \%$ & $68.98 \%$ \\
& $(z=4.52 ; p<.001)$ & $(z=6.44 ; p<.001)$ & $(z=3.79 ; p<.001)$ \\
B06 & $78.24 \%$ & $61.38 \%$ & $53.54 \%$ \\
& $(z=5.65 ; p<.001)$ & $(z=2.28 ; p<.05)$ & $(z=0.71 ; p<.47)$ \\
B08 & $70.80 \%$ & $76.08 \%$ & $60.48 \%$ \\
& $(z=4.16 ; p<.001)$ & $(z=5.21 ; p<.001)$ & $(z=2.1 ; p<.05)$ \\
\hline
\end{tabular}

Note $-z$ and $p$ values derive from one-tailed individual binomial tests comparing performance with chance $(50 \%)$ level. FO, first-order trials; SOI, secondorder increased trials; SOR, second-order reduced trials.

Both analyses (early or late baseline) converged to the same conclusion - that the lower performance with the probe trials was not due to an overtraining with the baseline stimuli. First, they showed a main effect of condition [early analysis, $F(3,12)=19.27, p<.001$; late analysis, $F(3,12)=25.11, p<.001]$. Duncan post hoc analyses indicated that the baseline trials yielded the highest performance $(p<.001)$. In addition, FO trials gave rise to greater performance than did SOR trials $(p<.05)$. There was also a tendency for higher scores in FO trials than in SOI trials, but this effect only approached significance $(p=.056$; see Figure 4$)$. Finally, there was no significant difference between SOI and SOR, suggesting that the distance separating the elemental features had no detectable effect on the processing of second-order relational cues. Because of this lack of difference between these two latter conditions, a one-way ANOVA was computed on the scores, with the test condition (baseline, FO, SO) as the unique within-subjects factor. This revealed a significant main effect of condition, with the following pattern or re-

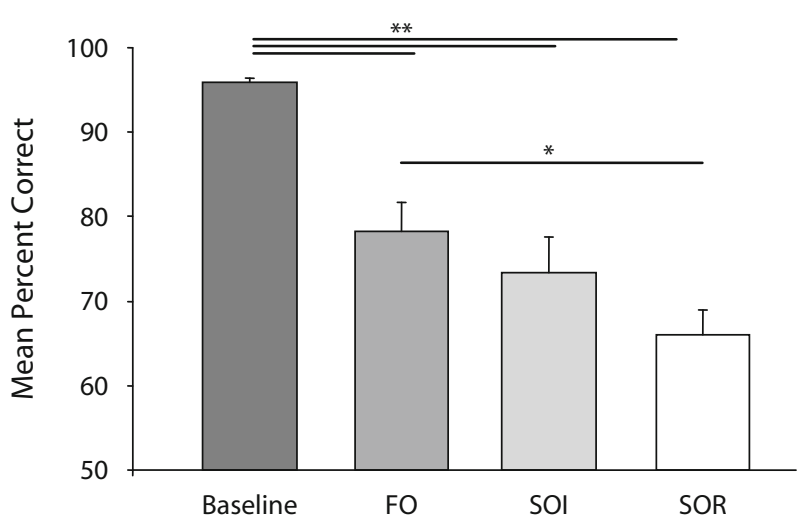

Figure 4. Mean percentage of correct responses (with standard errors) obtained, with each pair, in the baseline (considering all the trials) and the first-order (FO) and second-order increased (SOI) and reduced (SOR) trials. ${ }^{*} p<.05 .{ }^{* *} p<.001$. sults: baseline $>\mathrm{FO}>\mathrm{SO}[F(2,8)=32.36, p<.001]$. Figure 5 illustrates the pattern of findings for all subjects, to show its consistency.

Both ANOVAs also revealed a reliable effect of stimulus identity [early analysis, $F(9,36)=2.38, p<.05$; late analysis, $F(9,36)=2.43, p<.05]$, which interacted with the test condition [early analysis, $F(2,708)=3.46$, $p<.001$; late analysis, $F(27,108)=3.44, p<.001]$. As Figure 5 shows, that interaction is explained by the fact that stimuli $\mathrm{C}$ and I did not show the same (baseline $>$ first order $>$ second order) pattern of results as the other stimuli did. Finally, no effect of session emerged from either analysis [early analysis, $F(5,20)=2.63, p>.05$; late analysis, $F(5,20)=2.59, p>.05]$, indicating that baboons did not improve their performance across the six test sessions.

RTs were also analyzed, but this analysis neglected the early versus late baseline distinction, as well as the sessions, because they showed no real effects on scores. A two-way baseline (all trials, FO, SOI, SOR) $\times$ stimulus identity (10 different $\mathrm{S}+\mathrm{s}$ ) ANOVA on RTs showed no reliable effect of test condition or stimulus identity $[F(3,12)=1.11, p=.38$, and $F(9,36)=0.99, p=.46$, respectively] and no reliable interaction between these two factors $[F(27,108)=0.87, p=.65]$.

\section{DISCUSSION}

The main finding of the present research is that baboons could correctly recognize $\mathrm{S}+$ in our three test conditions (FO, SOI, and SOR), and, thus, that they are able to process both first- and second-order relational properties within nonface stimuli. Baboons also showed a tendency to prioritize first-order relational properties in configural processing, as revealed by a greater performance in these trials. Several reports of the literature converge with this conclusion. Parron and Fagot (2007a), for instance, found that baboons succeeded in the discrimination of a normal face from its first-order variant, but failed to spontaneously discriminate a normal face from its second- 


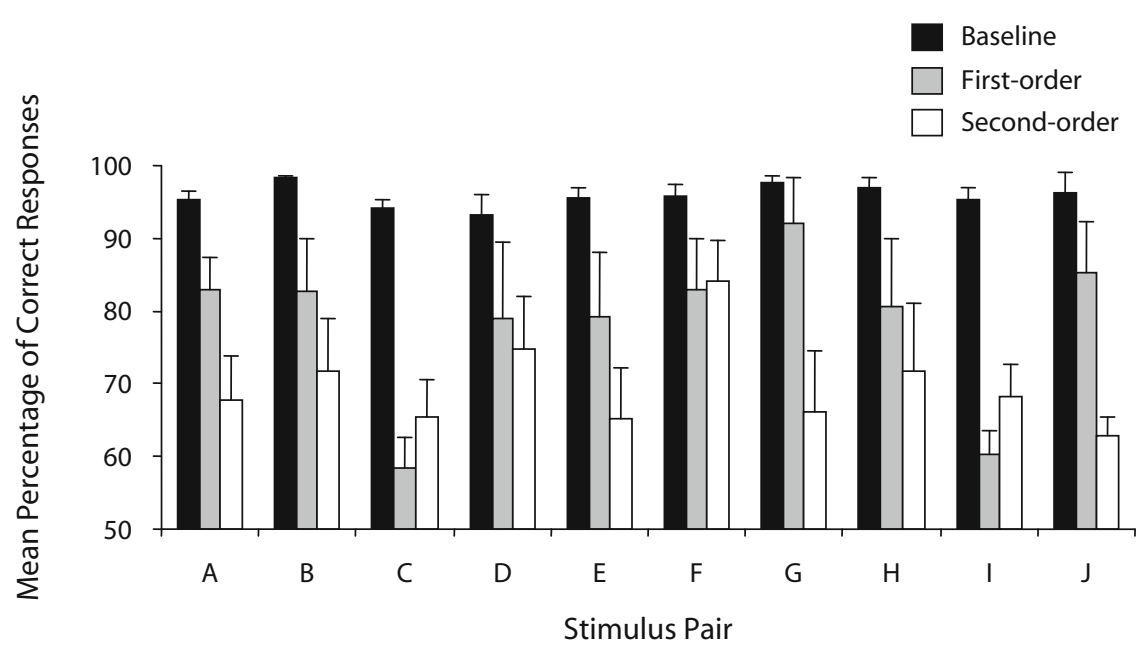

Figure 5. Mean percentages of correct responses (with standard errors) obtained in the test sessions in baseline, first-order, and second-order trials (averaged across the $10 \mathrm{~S}+$ greebles).

order modified variant. In another study, long-tailed macaques were impaired in the recognition of jumbled faces in which first-order properties have been disorganized (Dittrich, 1990). An infant gibbon, tested in a facelike versus nonface-like (first-order modified version) drawing-discrimination task, also showed a clear preference for the normal schematic faces (Myowa-Yamakoshi \& Tomonaga, 2001). Altogether, these convergent findings suggest that nonhuman primates pay particular attention to the first-order cues during the processing of objects with configural properties. In natural settings, analysis of first-order configural properties may have the adaptive function of allowing animals to quickly discriminate facial from nonfacial stimuli and, therefore, to potentially discriminate animals from nonanimals.

One noticeable difference between the present and previous studies is that the baboons were able to process the second-order relational properties within nonface stimuli, whereas before they were at chance level for second-order discrimination problems using faces (Parron \& Fagot, 2007a). We propose two main hypotheses to explain these discrepancies.

The first considers the inherent physical properties of the greeble stimuli. Facial stimuli convey many features, including the eyes, nose, and mouth, whereas our greebles contained only two distinctive features. The point can therefore be made that configural treatments were favored in our task by the request to process a reduced number of features to extract configural cues, rather than a complex combination of multiple features (a mechanism that is also suggested by the facilitation of configural processing, along with training; see, e.g., Gauthier \& Tarr, 1997). In addition, use of greebles, which are 3-D rendered stimuli, might have strengthened the saliency of an additional stimulus dimension, the depth. This dimension, probably not as salient in the case of faces, may have also facilitated the perception of the spatial relationships and, thus, the extraction of configural cues (Tsutsumi, Kikuchi, \& Nakajima, 1998).
An alternative, but nonexclusive, hypothesis for the emergence of configural processing with the greebles is that the extensive practice baboons received during training $(4,920$ trials altogether, on average, for the 10 pairs of greebles) enhanced the extraction of the second-order relational properties. Diamond and Carey (1986) proposed that configural processing can potentially apply to all objects, including faces, as a consequence of increasing expertise with these objects. In line with this idea, some studies have already shown that individualization requiring the processing of second-order configural cues occurs mainly with the faces of conspecifics (Dahl, Logothetis, \& Hoffman, 2007; Humphrey, 1974; Parr et al., 2006) - that is, for which the subjects have expertise. By contrast, monkeys tend to use only feature-based strategies to recognize human (or unfamiliar) faces for which they have less expertise (e.g., Gothard, Brooks, \& Peterson, 2009). There is, moreover, an additional procedural difficulty when configural processing is investigated in monkeys with pictures of faces. Serious doubts can be drawn from the fact that pictorial facial stimuli were systematically processed as faces per se in previous monkey experiments (e.g., Martin-Malivel \& Fagot, 2001; Parron \& Fagot, 2007a). Even if we admit that the perception of real faces might promote configural processing in monkeys, due to a daily acquired expertise with faces, that expertise might not systematically transfer to 2-D pictorial faces. Because (1) expertise specifically promotes the processing of second-order properties (in humans: Gauthier \& Tarr, 1997; in monkeys: Gauthier \& Logothetis, 2000; Parr \& Heintz, 2008; in chimpanzees: Parr et al., 1998) and (2) pictorial faces might be processed as novel meaningless visual patterns rather than as meaningful real faces (see above), it can be predicted that expertise effects do not systematically apply and generalize from the real world when pictures of faces are used as stimuli, therefore hampering the demonstration of second-order configural processing in studies of face recognition in nonhuman primates. 
Another goal of our study was to assess whether reliance on second-order cues is scale dependent. Our results showed that baboons performed similarly well with an increased distance between the features in the SOI and SOR trials, in which the distance between the features was either increased or reduced. This lack of difference may, at first glance, appear at odds with the demonstration that baboons have difficulties in grouping elements togetherin particular, when the distance between these elements is large (Fagot \& Deruelle, 1997; Parron \& Fagot, 2007b; Spinozzi, De Lillo, \& Salvi, 2006). However, these early experiments on perceptual grouping used stimuli made of spatially distinct elemental features, whereas the present research involved configural changes within spatially continuous stimuli. As proposed by Gestalt principles (e.g., Koffka, 1935), one of the most fundamental perceptual grouping principles is proximity, implying that nearby objects tend to be perceived as belonging to a common group. We, therefore, propose that the processing of the fine spatial relations among features in continuous stimuli does not share the nature of perceptual grouping for spatially disjoint elements. Irrespective of this issue, absence of a reliable difference between the SOI and SOR condition suggests that the processing of second-order cues in monkeys is scale independent.

In summary, this study demonstrates that baboons can process the configural properties of stimuli, even if they are not faces. It moreover supports the idea that, in monkeys, the processing of configural relations is subject to extensive training. The few previous investigations on this issue have almost systematically adopted a neuroscientific approach that considered the neuronal selectivity of the visual system. In agreement with our findings, these studies suggest that a homogeneous class of nonface objects, for which the monkeys have been extensively trained, can induce configurational selectivity in the neuronal response of the visual system (e.g., Gauthier \& Logothetis, 2000; Logothetis \& Pauls, 1995; Logothetis, Pauls, \& Poggio, 1995). Our findings are also in accordance with the literature of human studies, suggesting that nonface categories of objects can be processed in the same way as faces, as a consequence of expertise (for a review, see Bukach et al., 2006). From a theoretical point of view, if face and nonface stimuli lead to qualitatively similar behavioral effects in recognition tasks, it is reasonable to postulate that a common mechanism could give rise to both. The configural sensitivity that baboons showed with the greebles suggests that face perception may be carried out by domaingeneral mechanisms in nonhuman primates - that is, by a mechanism not uniquely specialized for face processing. That mechanism may have an adaptive function and may facilitate the discrimination of highly similar examplars of objects belonging to the same category, considering their second-order configural properties.

\section{AUTHOR NOTE}

C.P. was supported by Fondation pour la Recherche Médicale Grant SPF20081215261. Development of the ALDM system was supported by a PACA "Volet exploratoire" Grant to J.F. Marc Martin is acknowledged for technical support. The procedure used in this research re- ceived an agreement from the "PACA Comité regional d'éthique pour l'expérimentation animale." Address correspondence to C. Parron, LPC, CNRS, UMR 6146 Université d'Aix-Marseille, 3 place Victor Hugo, Bat.9, Case D, 13331 Marseille Cedex, France (e-mail: carole.parron@, univ-provence.fr).

\section{REFERENCES}

BRUCE, C. (1982). Face recognition by monkeys: Absence of an inversion effect. Neuropsychologia, 20, 515-522.

Bukach, C. M., Gauthier, I., \& TARr, M. J. (2006). Beyond faces and modularity: The power of an expertise framework. Trends in Cognitive Sciences, 10, 159-166.

Dahl, C. D., Logothetis, N. K., \& Hoffman, K. L. (2007). Individuation and holistic processing of faces in rhesus monkeys. Proceedings of the Royal Society B, 274, 2069-2076.

Deruelle, C., Barbet, I., Dépy, D., \& Fagot, J. (2000). Perception of partly occluded figures by baboons (Papio papio). Perception, 29, 1483-1497.

DiAmond, R., \& CAREY, S. (1986). Why faces are and are not special: An effect of expertise. Journal of Experimental Psychology: General, 115, 107-117.

DitTRICH, W. (1990). Representation of faces in longtailed macaques (Macaca fascicularis). Ethology, 85, 265-278.

Fagot, J., Bonté, E., \& Parron, C. (2009). Concept of uprightness in baboons: Assessment with pictures of realistic scenes. Animal Cognition, 12, 369-379. doi:10.1007/s10071-008-0196-6

FAGOT, J., \& Deruelle, C. (1997). Processing of global and local visual information and hemispheric specialization in humans (Homo sapiens) and baboons (Papio papio). Journal of Experimental Psychology: Human Perception \& Performance, 23, 429-442.

Fagot, J., \& Paleressompoulle, D. (2009). Automatic testing of cognitive performance in baboons maintained in social groups. Behavior Research Methods, 41, 396-404. doi:10.3758/BRM.41.2.396

FAGOT, J., \& PARRon, C. (2010). Relational matching in baboons with reduced grouping requirements. Journal of Experimental Psychology: Animal Behavior Processes, 36, 184-193.

Gauthier, I., \& Logothetis, N. K. (2000). Is face recognition not so unique after all? Cognitive Neuropsychology, 17, 125-142.

Gauthier, I., \& TARr, M. J. (1997). Becoming a "greeble" expert: Exploring mechanisms for face recognition. Vision Research, 37, 16731682.

Gauthier, I., Williams, P., Tarr, M. J., \& Tanaka, J. (1998). Training "greeble" experts: A framework for studying expert object recognition processes. Vision Research, 38, 2401-2428.

Gothard, K. M., Brooks, K. N., \& Peterson, M. A. (2009). Multiple perceptual strategies used by macaque monkeys for face recognition. Animal Cognition, 12, 155-167. doi:10.1007/s10071-008-0179-7

Humphrey, N. K. (1974). Species and individuals in the perceptual world of monkeys. Perception, 3, 105-114. doi:10.1068/p030105

KoffKa, K. (1935). Principles of Gestalt psychology. New York: Harcourt, Brace.

Logothetis, N. K., \& Pauls, J. (1995). Psychophysical and physiological evidence for viewer-centered object representations in the primate. Cerebral Cortex, 5, 270-288. doi:10.1093/cercor/5.3.270

Logothetis, N. K., Pauls, J., \& Poggio, T. (1995). Shape representation in the inferior temporal cortex of monkeys. Current Biology, 5, 552-563.

Martin-Malivel, J., \& Fagot, J. (2001). Perception of pictorial human faces by baboons: Effects of stimulus orientation on discrimination performance. Animal Learning \& Behavior, 29, 10-20.

Maurer, D., Le Grand, R., \& Mondloch, C. J. (2002). The many faces of configural processing. Trends in Cognitive Sciences, 6, 255260.

Myowa-Yamakoshi, M., \& Tomonaga, M. (2001). Development of face recognition in an infant gibbon (Hylobates agilis). Infant Behavior \& Development, 24, 215-227. doi:10.1016/S0163-6383(01)00076-5

Parr, L. A., Dove, T., \& Hopkins, W. D. (1998). Why faces may be special: Evidence of the inversion effect in chimpanzees. Journal of Cognitive Neuroscience, 10, 615-622.

Parr, L. A., \& Heintz, M. (2008). Discrimination of faces and houses by rhesus monkeys: The role of stimulus expertise and rotation angle. Animal Cognition, 11, 467-474. doi:10.1007/s10071-008-0137-4 
Parr, L. A., Heintz, M., \& Akamagwuna, U. (2006). Three studies on configural face processing by chimpanzees. Brain \& Cognition, 62, 30-42. doi: 10.1016/j.bandc.2006.03.006

Parr, L. A., Heintz, M., \& Pradhan, G. (2008). Rhesus monkeys (Macaca mulatta) lack expertise in face processing. Journal of Comparative Psychology, 122, 390-402. doi:10.1037/0735 $-7036.122 .4 .390$

Parron, C., \& Fagot, J. (2007a). Baboons (Papio papio) spontaneously process the first-order but not second-order configural properties of faces. American Journal of Primatology, 70, 415-422. doi:10.1002/ ajp.20503

Parron, C., \& FAGOT, J. (2007b). Comparative of grouping abilities in humans (Homo sapiens) and baboons (Papio papio) with the Ebbinghaus illusion. Journal of Comparative Psychology, 121, 405-411. doi:10.1037/0735-7036.121.4.405

Parron, C., \& Washburn, D. (2010). Contrasting the edge- and the surface-based theories of object recognition: Behavioral evidence from macaques (Macaca mulatta). Journal of Experimental Psychology: Animal Behavior Processes, 36, 148-157. doi:10.1037/a0015629

Rhodes, G., \& McLean, I. G. (1990). Distinctiveness and expertise effects with homogeneous stimuli: Towards a model of configural coding. Perception, 19, 773-794. doi:10.1068/p190773

Rosenfeld, S. A., \& VAN Hoesen, G. W. (1979). Face recognition in the rhesus monkey. Neuropsychologia, 17, 503-509.
Spinozzi, G., De Lillo, C., \& SAlvi, V. (2006). Local advantage in the visual processing of hierarchical stimuli following manipulations of stimulus size and element numerosity in monkeys (Cebus apella). Behavioural Brain Research, 166, 145-154. doi:10.1016/j.bbr.2005.06.043

Thompson, P. (1980). Margaret Thatcher: A new illusion. Perception, 9, 483-484.

Tomonaga, M. (1994). How laboratory-raised Japanese monkeys (Macaca fuscata) perceive rotated photographs of monkeys: Evidence for an inversion effect in face perception. Primates, 35, 155165. doi:10.1007/BF02382051

Tomonaga, M., Itakura, S., \& Matsuzawa, T. (1993). Superiority of conspecific faces and reduced inversion effect in face perception by a chimpanzee. Folia Primatologica, 61, 110-114.

Tsutsumi, S., KikUChI, S., \& NaKaJima, M. (1998). Face identification using a 3D gray-scale image: A method for lessening restrictions on facial directions. In Proceedings of the 3rd IEEE International Conference on Automatic Face and Gesture Recognition (pp. 306-311). Los Alamitos, CA: IEEE Computer Society Press.

YIN, R. K. (1969). Looking at upside-down faces. Journal of Experimental Psychology, 81, 141-145.

(Manuscript received February 2, 2010; accepted for publication March 16, 2010.) 